KOZAK, A. 1962. Sequential Sampling of Megastigmus Spermotrophus (Wachtl) Infestation on Douglas Fir. Unpublished repore for Professor J. H. G. Smith, Faculty of Forestry, U.B.C.

McCRACKEN, and D. DANIEL. 1961. A Guide to FORTRAN Programming. John Wiley and Sons, New York.

SMITH J. H. G. and J. W. Ker. 1957. Some Distributions Encountered in Sampling Forest Stands. For. Sc. Vol. 3. No. 2 pp. 137-143.

STEEL, G. D. and J. H. TORRIE, 1960. Principles and Procedures of Statistics. McGraw-Hill Book Co. Inc. New York.

\title{
Table of Log Volume Factors
}

By C. Joergensen ${ }^{1}$

The following factors represent volume, in cubic feet, per foot of log length; hence the volume of a log equals its length times the appropriate factor, as determined from the two end-diameters.

The volume factor for a given $\log$ is found in the row corresponding to smallest diameter $(\mathrm{d})$ of the $\log$, and in the column corresponding to the difference (D - d) between largest diameter (D) and smallest diameter.

EXAMPLE:

$\begin{array}{lc}\text { largest diameter }(D) & 20 \text { inches } \\ \text { smallest diameter }(d) & 16 \text { inches } \\ \text { difference }(D-d) & 4 \text { inches } \\ \text { length }(L) & 32 \text { feet } \\ \text { factor }(\text { row } 16, \text { col. } 4) & 1.789 \\ \text { volume }(1.789 \times 32) & 57.248 \mathrm{cu} . \mathrm{ft} .\end{array}$

Attention is drawn to the term smallest diameter, which must be used as index to rows, regardless of whether it happens to be the top or the butt diameter. Normally, top diameter is smaller than butt diameter; but this may be reversed, for example, by a defect deduction at the butt end.

The formula used to calculate the volume factors $(v)$ is a transposed version of the Smalian formula (length $=1$ foot) :

$$
v=\left(\mathrm{d}^{2}+\mathrm{D}^{2}\right) \times 0.00272707696
$$

Log volumes calculated by the factor method are identical with those obtained from the authorized Table of Ten Times Volume of Cylinders in Cubic Feet. 


\begin{tabular}{|c|c|c|c|c|c|c|c|c|c|c|c|c|}
\hline & 0 & 1 & 2 & 3 & 4 & 5 & 6 & 7 & 8 & 9 & 10 & 11 \\
\hline 0 & 0.000 & 0.003 & $0.01 \mathrm{II}$ & 0.024 & 0.044 & 0.068 & 0.098 & 0.134 & 0.174 & 0.221 & 0.273 & $0.33^{\circ}$ \\
\hline 1 & 0.005 & 0.014 & 0.027 & 0.0 .46 & $0.07 i$ & 0.101 & $0.13^{6}$ & 0.177 & 0.224 & 0.275 & 0.333 & 0.395 \\
\hline 2 & 0.022 & 0.035 & 0.055 & 0.079 & 0.109 & 0.145 & 0.185 & 0.232 & 0.284 & $0.34 \mathrm{I}$ & 0.404 & 0.472 \\
\hline 3 & 0.049 & 0.068 & 0.093 & 0.123 & $0.15^{8}$ & 0.199 & 0.245 & 0.297 & 0.355 & 0.417 & 0.485 & 0.559 \\
\hline 4 & 0.087 & 0.112 & 0.142 & 0.177 & 0.218 & 0.265 & 0.316 & 0.374 & 0.436 & 0.505 & 0.578 & 0.657 \\
\hline 5 & 0.136 & 0.166 & 0.202 & 0.243 & 0.289 & $0.34 \mathrm{I}$ & $0.39^{8}$ & 0.461 & 0.529 & 0.6003 & 0.682 & 0.766 \\
\hline 6 & 0.196 & $0.23^{2}$ & 0.273 & 0.319 & $0.37^{1}$ & 0.428 & 0.491 & 0.559 & 0.633 & 0.712 & 0.796 & 0.886 \\
\hline 7 & 0.267 & 0.308 & 0.355 & 0.406 & 0.464 & 0.526 & 0.595 & 0.668 & 0.747 & 0.832 & 0.922 & 1.017 \\
\hline 8 & 0.349 & 0.395 & 0.447 & 0.505 & 0.567 & 0.635 & 0.709 & 0.788 & 0.873 & 0.963 & 1.058 & I.159 \\
\hline 9 & $0.44^{2}$ & 0.494 & 0.551 & 0.614 & 0.682 & 0.755 & 0.834 & 0.919 & $1 . \infty 09$ & $\mathrm{I}_{\mathrm{IO}}$ & 1.205 & 1.312 \\
\hline 10 & 0.545 & 0.603 & 0.665 & 0.734 & 0.807 & 0.886 & $0.97 !$ & $1.06 \mathrm{I}$ & 1.156 & 1.257 & I. 364 & 1.475 \\
\hline 11 & 0.660 & 0.723 & $0.79 \mathrm{I}$ & $0.86_{4}$ & 0.944 & 1.028 & I.118 & $I .2 I_{4}$ & $1.3 I_{4}$ & $1.4^{2 \mathrm{r}}$ & 1.533 & 1.650 \\
\hline 12 & 0.785 & 0.854 & 0.927 & 1.006 & 1.091 & $x .181$ & I. .276 & I. 377 & 1.484 & 1.595 & 1.713 & 1.835 \\
\hline 13 & 0.922 & 0.995 & 1.074 & 1.159 & 1.249 & 1.344 & $I .445$ & 1.552 & $1.66_{4}$ & 1.781 & 1.903 & 2.031 \\
\hline 14 & 1.069 & 1.148 & 1.233 & 1.323 & 1.418 & 1.519 & 1.625 & 1.737 & 1.854 & โ. 977 & 2105 & 2.239 \\
\hline 15 & I. 227 & 1.312 & 1.402 & J.497 & $1.59^{8}$ & $1.70_{4}$ & 1.816 & I.933 & 2.056 & 2.184 & 2.318 & 2.457 \\
\hline 16 & 1.396 & 1.486 & T. 582 & $1.68 \mathrm{~J}$ & 1.789 & 1.901 & 2.018 & $2 . I_{4} I$ & 2.269 & 2.403 & 2.542 & 2.686 \\
\hline 17 & 1.576 & 1.672 & 1.773 & $\mathrm{~J} .879$ & 1.991 & 2.108 & 1.231 & 2.359 & 2.493 & 2.632 & $2.77^{6}$ & 2.926 \\
\hline 18 & I. 767 & 1.868 & 1.974 & 2.086 & 2.203 & 2.326 & 2.454 & 2.588 & 2.727 & 2.872 & 3.022 & 3.177 \\
\hline 19 & 1.969 & $=.075$ & 2.187 & 2.304 & 2.427 & $=2.555$ & 2.689 & $2.8 \approx 8$ & 2.973 & 3.123 & $3.27^{8}$ & 3.439 \\
\hline 20 & 2.182 & 2.293 & 2.411 & 2.533 & 2.662 & 2.795 & 2.934 & 3.079 & 3.229 & $3 \cdot 3^{84}$ & 3.545 & 3.712 \\
\hline 21 & 2.405 & $2.5 \div 3$ & 2.645 & 2.773 & 2.907 & 3.046 & $3.19 \mathrm{I}$ & 3.341 & 3.496 & 3.657 & $3.8=3$ & 3.995 \\
\hline 22 & $=.640$ & 2.763 & $2.89 \mathrm{I}$ & 3.024 & 3.163 & 3.308 & $3.45^{8}$ & 3.613 & 3.774 & $3.94 \mathrm{I}$ & 4.112 & 4.290 \\
\hline 23 & 2.885 & 3.013 & 3.147 & 3.286 & $3 \cdot 43^{1}$ & $3.58 \mathrm{I}$ & 3.736 & 3.897 & 4.063 & 4.235 & +412 & +.595 \\
\hline 24 & 3.142 & 3.275 & $3 \cdot 4^{1} 4$ & 3.559 & 3.709 & 3.864 & 4.025 & 4.192 & 4.363 & $4 \cdot \S 4 \mathrm{I}$ & 4.723 & 4.911 \\
\hline 25 & 3.409 & $3.54^{8}$ & 3.692 & 3.842 & 3.998 & 4.159 & 4.325 & 4.497 & 4.674 & +857 & 5.045 & 5.239 \\
\hline 26 & & 3.832 & & 4. I 37 & 4.29 & 4.4 & & 4.813 & 4.996 & 5.184 & 5.378 & 5.577 \\
\hline 27 & 3.976 & 4.126 & 4.282 & $4 \cdot 44^{2}$ & 4.609 & 4.781 & 4.958 & $5.14 \mathrm{I}$ & 5.329 & 5.522 & 5.721 & 5.926 \\
\hline 28 & $4-276$ & $4 \cdot 43=$ & $4.59=$ & 4.759 & 4.931 & 5.108 & 5.291 & 5.479 & 5.672 & 5.871 & 6.076 & 6.286 \\
\hline 29 & $4.5^{87}$ & $4.74^{8}$ & $4 \cdot 9^{1} 4$ & 5.086 & 5.263 & $5 \cdot 4+6$ & 5.634 & $5.8=8$ & 6.027 & 6.231 & $6.44^{1}$ & 6.657 \\
\hline 30 & 4.909 & 5.075 & 5.247 & $5 \cdot 4^{2} 4$ & 5.607 & 5.795 & $5.9^{89}$ & 6.188 & 6.392 & 6.602 & 6.818 & 7.039 \\
\hline 31 & $5.24 \mathrm{I}$ & 5.413 & 5.591 & 5.773 & 5.961 & 6.155 & 6.35 & 6.559 & 6.769 & 6.984 & 7.205 & $7 \cdot 431$ \\
\hline 32 & $5.5^{8} 5$ & 5.762 & 5.945 & 6.133 & 6.327 & $6.5 \geq 6$ & $6.73^{\circ}$ & 6.940 & 7.756 & 7.377 & 7.603 & 7.835 \\
\hline 33 & $5.94^{\circ}$ & 6.122 & 6.310 & 6.504 & 6.703 & 6.908 & 7.118 & 7.333 & 7.554 & 7.780 & 8.012 & 8.249 \\
\hline 34 & 6.305 & 6.493 & 6.687 & 6.886 & 7.090 & 7.300 & 7.516 & 7.737 & 7.963 & 8.195 & $8.43^{2}$ & 8.675 \\
\hline 35 & 6.681 & 6.875 & 7.074 & 7.279 & $7.4^{89}$ & 7.704 & 7.925 & $8.15 \mathrm{I}$ & $8.3^{8} 3$ & 8.620 & 8.863 & 9.111 \\
\hline 36 & 7.069 & 7.268 & $7 \cdot 472$ & & 7.898 & 8.119 & 8.345 & 8.577 & $8.8 \mathrm{1}_{4}$ & 9.057 & 9.305 & $9.55^{8}$ \\
\hline 37 & 7.467 & 7.671 & 7.881 & 8.097 & 8.318 & 8.544 & 8.7 & 9.013 & 9.2 & & 9.757 & 10.017 \\
\hline 38 & 7.876 & 8.086 & 8.301 & 8.522 & 8.748 & 8.980 & 9.218 & 9.460 & 9.708 & 9.962 & 10.221 & $10.4^{86}$ \\
\hline 39 & 8.296 & 8.531 & 8.732 & 8.958 & 9.190 & 9.428 & 9.670 & 9.918 & 10.172 & 10.431 & 10.696 & 10.966 \\
\hline 40 & 8.727 & $8.94^{8}$ & 9.174 & 9.406 & 9.643 & 9.886 & 10.134 & 10.387 & 10.647 & 10.9 II & 11.181 & 11.456 \\
\hline 41 & 9.168 & 9.395 & 9.627 & $9.86_{4}$ & 10.107 & 10.355 & 10.608 & 10.867 & JI.132 & II.4OI & 11.677 & $11.95^{8}$ \\
\hline 42 & & 9.853 & $10.09^{\circ}$ & 10.333 & & 10.835 & 11.094 & $11.35^{8}$ & 11.628 & & 12.185 & {$[2.47]$} \\
\hline 43 & 10.085 & 10.322 & 10.565 & $10.8 \mathrm{I} 3$ & 11.066 & 11.326 & 11.590 & 11.860 & 12.135 & 52.416 & 12.703 & 12.995 \\
\hline 44 & 10.559 & 10.802 & 11.050 & 11.304 & 11.563 & I 1.827 & 12.097 & 12.373 & 12.654 & $12.94^{\circ}$ & 13.2 .32 & 13.529 \\
\hline 45 & 11.045 & 11.293 & $11.54^{6}$ & โ1.806 & 12.07 & 12.340 & 12.615 & 12.896 & 13.183 & & 13.772 & 14.074 \\
\hline 46 & & 1.795 & 12.054 & & 12.588 & 12.864 & 13.145 & 13.431 & 13.723 & & & $4.63 \mathrm{i}$ \\
\hline 47 & & 12.307 & $12.57^{2}$ & 12.842 & 13.117 & ${ }^{1} 3.39^{8}$ & I 3.684 & $\times 3.976$ & & 14.576 & $14.88_{4}$ & $15.19^{\circ}$ \\
\hline 48 & 12.566 & 12.831 & 13.10I & 13.376 & 13.657 & I 3.944 & $\mathbf{I}_{4} .235$ & 1.533 & 1.835 & 15.143 & 15.457 & 15.776 \\
\hline 49 & 13.095 & 13.365 & $13.64 \mathrm{I}$ & 13.922 & 14.208 & I 4.500 & 14.797 & 15.100 & 15.408 & 15.722 & $16.0_{4}$, & 16.365 \\
\hline 50 & 13.635 & $13.9^{1 \mathrm{I}}$ & 14.192 & 54.478 & $14.77^{\circ}$ & 15.067 & $15.37^{\circ}$ & $15.67^{8}$ & -15.992 & 16.311 & 16.635 & 16.965 \\
\hline
\end{tabular}

